\title{
Conceptualizaciones, perspectivas y referentes de la participación en la educación primaria, México
}

Denys Serrano-Arenas, Ph.D.

Posdoctorante Universidad Autónoma de

Querétaro, México*

Evaristo Arcos-Miranda, Ph.D.

Profesor Universidad Abierta y a Distancia de

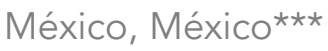

dserrano837@gmail.com

\author{
Azucena Ochoa-Cervantes, Ph.D. \\ Profesora Universidad Autónoma de Querétaro, \\ México**
}

\section{Resumen (analítico)}

Se analizan las conceptualizaciones sobre participación que refieren niñas y niños (entre cinco y ocho años de edad), así como las de sus profesores y familiares, en tres escuelas de educación primaria de zonas urbano-marginales en México. El método cuasietnográfico fue utilizado para describir e interpretar los significados obtenidos de las entrevistas individuales, grupos focales y el dibujo infantil. La información fue analizada por medio del análisis de contenido categorial. Los resultados muestran que, conforme la infancia avanza en su escolarización, los significados sobre participación se acotan a emitir opiniones cuando le son solicitadas por los profesores. Se identifica al adultocentrismo y obediencia como los obstáculos de la construcción de escuelas inclusivas, concluyendo que es imperativo generar diálogos entre los referentes conceptuales sobre participación provenientes del espacio familiar y educativo.

Palabras clave

Conceptualización, participación, infancia, inclusión.

\section{Tesauro}

Tesauro de Ciencias Sociales de la Unesco y Tesauro Eric.
Para citar este artículo

Serrano-Arenas, D., Ochoa-Cervantes, A., \& Arcos-Miranda, E. (2019). Conceptualizaciones, perspectivas y referentes de la participación en la educación primaria, México. Revista Latinoamericana de Ciencias Sociales, Niñez y Juventud, 17(2), 1-22. doi:10.11600/1692715x.17202

Historial

Recibido: 06.02.2019

Aceptado: 26.04.2019

Publicado: 30.06.2019

Información artículo

Este artículo forma parte del proyecto denominado «Promoción de la participación de la infancia en el contexto escolar», realizado del 30 de julio al 3 de diciembre del 2018. El financiamiento de la investigación se otorgó por el Consejo Nacional de Ciencia y Tecnología (Conacyt), con número de solicitud 2018-000005-01NACV-00368, adscrito en la Universidad Autónoma de Querétaro. Área: Sociología. Subárea: etnografía. 


\section{Conceptualizations, perspectives and reference points for participation in elementary schools, Mexico}

Abstract (analytical)

This paper reviews the conceptualizations of participation among children aged between five and eight years old, their teachers and their family members in three elementary schools in urban marginalized areas of Mexico. A quasi-ethnographic method was used for the description and interpretation of the meanings identified in individual interviews, focal groups and children's drawings. The information was reviewed using a process involving categorical content analysis. The results indicate that as the children progress through their schooling process, the meanings related to participation are limited to issuing opinions when requested by teachers. Adult-centrism and obedience norms are identified as obstacles that limit the construction of inclusive schools. The authors conclude that it is imperative to generate dialogues between the conceptual reference points about child participation in family and educational spaces.

Keywords

Conceptualization, participation, childhood, inclusion.

\section{Conceitos, perspectivas e referências da participação na educação primária no México}

Resumo (analítico)

Neste trabalho são analisados conceitos sobre a participação de crianças, entre cinco e oito anos de idade, e a participação de professores e familiares em três escolas de Ensino Fundamental de áreas urbanas marginalizadas do México. O método quase etnográfico foi utilizado para descrever e interpretar os significados a partir de entrevistas individuais e grupais, bem como de desenhos infantis. A informação foi estudada por meio de uma Análise de conteúdo categorial. Os resultados mostram que conforme as crianças avançam na sua escolaridade, os resultados sobre participação se limitam a emitir opiniões quando lhe são solicitadas pelos professores no espaço escolar. O centrismo adulto e a obediência são identificados como obstáculos à construção de escolas inclusivas, concluindo que é imprescindível gerar diálogos entre os referentes conceituais sobre a participação proveniente da família e do espaço educacional.

Palavras-chave

Conceitualização, participação, infância e educação inclusiva.

\section{Información autores}

[*] Licenciada en Educación Física. Maestra en Procesos Educativos (Universidad Autónoma Chapingo). Doctora en Ciencias en Educación Agrícola Superior (Universidad Autónoma de Chapingo). Posdoctorante en el programa de maestría Educación para la Ciudadanía. Orcid: 0000-0002-4379-8863. Índice H5: 1. Correo electrónico:

dserrano837@gmail.com

[**] Licenciada en Pedagogía. Maestra en Psicología Educativa (Universidad Autónoma de Querétaro). Doctora en Psicología y Educación (Universidad Autónoma de Querétaro). Posdoctorado en la Universidad de Alicante. Orcid: 0000-0003-4515-9069. Índice H5: 2. Correo electrónico: azus@uaq.mx

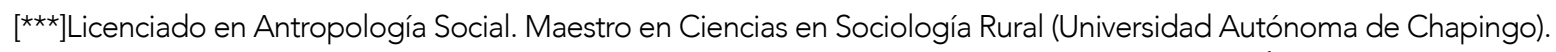
Doctor en Ciencias Agrarias (Universidad Autónoma de Chapingo). Orcid: 0000-0002-2474-9046. Índice H5: 1. Correo electrónico: clandestino_7manonegra@yahoo.com.mx 


\section{Introducción}

En la actualidad, los fundamentos de las escuelas inclusivas no solo se enfocan en el estudio de niños y niñas con necesidades educativas especiales, sino también priorizan el estudio de una organización escolar que reconozca la diversidad y brinde respuestas a las múltiples necesidades que coexisten en los espacios educativos (Ainscow, 2012). La participación, al permitir la expresión, la toma de decisiones y el diálogo horizontal, genera múltiples entendimientos entre niñas, niños y adultos; estas expresiones brindan reconocimiento a los sujetos implicados, lo que lleva a las personas a sentirse valoradas y parte de la comunidad escolar, por lo que la participación se convierte en un eje fundamental de la inclusión.

Por esto, la participación de la infancia es un área de interés en las políticas e investigación educativa al constituir un indicador de la inclusión y justicia. No obstante, si bien hay un consenso sobre su importancia, no lo hay sobre su conceptualización. Existen diversas perspectivas que delinean las conceptualizaciones y los fundamentos del estudio de la participación, entre las que se destacan las siguientes.

La perspectiva política hace referencia a la participación como un derecho en relación con la expresión y la toma de decisiones en todo proceso que afecte a la niñez. Por su parte, la perspectiva pedagógica aborda la participación como un proceso educativo que permite el ejercicio de la ciudadanía desde la infancia (Mendia, 2017; Ochoa-Cervantes, 2015). Por otro lado, la perspectiva social se centra en los marcos de acción de los sujetos, retomando a niños y niñas como actores sociales (Alderson, 2015; Arce, 2015; Liebel \& Invernizzi, 2018; Miranda \& Albarrán, 2019; Stoecklin, 2013), mientras que la perspectiva culturalista destaca los significados que construyen los sujetos sobre su participación a partir de su contexto (Frasco-Zuker, 2016; Hedrera-Manara \& Álvarez-Martínez, 2018; LeyraFatou, \& Bárcenas-Viñas, 2014; Martínez-Núñez, \& Muñoz-Zamora, 2015; Valle, 2018).

Las anteriores miradas dan cuenta de que el abordaje de la participación en los entornos educativos es diverso, por lo que las prácticas participativas a las que la infancia 
tiene acceso dependen de los referentes conceptuales de su medio sociocultural. Por lo anterior, es menester generar un diálogo entre las conceptualizaciones de participación de niños y niñas con aquellas propias de los profesores y familiares, a fin de reflexionar sobre los objetivos de la participación que se pretende promover. De lo contrario, se corre el riesgo de caer en prácticas participativas tradicionales que promueven el adultocentrismo, agravando los problemas de desigualdad y exclusión escolar.

Ante esta necesidad, en varias investigaciones se han analizado los constructos conceptuales de los actores implicados en espacios educativos (infancia, adolescencia, autoridades escolares, profesores y familiares) (Albornoz, Silva, \& López, 2015; CarrascoAguilar et al., 2016; Moreno-Roldán, Agudelo-Bedoya \& Alzate-Pulgarín, 2018; Thomas, Graham, Powell, \& Fitzgerald, 2016). Entre los estudios enfocados en el análisis de las conceptualizaciones de la infancia sobresale el de Ochoa-Cervantes (2015), quien expresa:

La concepción que muestran los niños, niñas y adolescentes encuestados en la presente investigación es limitada, ya que la participación se reduce a la emisión de una opinión, mostrando con esto que la participación que se promueve en la escuela es una participación simple o en el mejor de los casos consultiva, pues dadas las respuestas de los participantes, por una parte el alumnado participa siguiendo indicaciones o respondiendo a estímulos, y por la otra, se les demanda una opinión, la cual deben emitir según las indicaciones que tienen bien interiorizadas. (p. 22)

Albornoz et al. (2015) analizan los significados de participación entre niños y niñas de once y doce años de edad, en dos categorías: ser escuchado y toma de decisiones. Se destaca que la infancia no considera que la tomen en cuenta en los procesos educativos, por lo que es imperativo reforzar el reconocimiento de la infancia en la construcción de su proceso de aprendizaje.

Estas investigaciones hacen referencia a una participación aprendida de manera jerárquica, en la que niños y niñas construyen sus conceptos y referentes acerca de la participación a partir de los adultos que los rodean. Dichos análisis, al solo considerar las concepciones de participación de niños y niñas mayores de ocho años, dejan los conceptos de la infancia del menor edad fuera del análisis, lo cual hace que esta etapa de la infancia sea una de las menos conocidas y exploradas.

La escuela primaria en México atiende a la población estudiantil en un rango de edad, principalmente entre seis y doce años, y organiza la atención escolar en seis grados. En este espacio educativo los obstáculos del ejercicio de su participación se agudizan con 
niños y niñas de menor edad, puesto que las representaciones socioculturales que poseen los adultos de los niños más pequeños recaen con mayor facilidad en el proteccionismo y la inocencia (Ancheta-Arrabal, 2015; Ancheta-Arrabal, 2019; Gascón \& Godoy, 2015; Giroux, 2003; Liebel, 2015; Voltarelli, 2017); ello debido a que se enfrentan con mayor frecuencia a una restricción al ejercer su participación, fundamentada en representaciones sociales que destacan sus carencias sobre lo que son capaces de pensar, elegir y hacer.

Aunado a lo anterior, los estudios sobre la infancia de menor edad (entre cuatro y ocho años) se han enfocado en la construcción de las conceptualizaciones de los docentes (Cubillos, Chiappe, González, \& Velásquez, 2018; Díaz-Bórquez, Contreras-Shats, \& Bozo-Carrillo, 2018; Gallego-Henao \& Gutiérrez-Suárez, 2015; Martínez-Núñez, \& Muñoz-Zamora, 2015; Patiño \& Danna, 2017). Al no considerar las conceptualizaciones de niños y niñas más pequeños, se complica la tarea de identificar los primeros referentes que abstraen y delinean el concepto de participación durante sus primeros años escolares; lo anterior dificulta el entendimiento de los retos que conlleva su ejercicio y el desarrollo de una perspectiva inclusiva de la participación que promueva su protagonismo y, por tanto, su injerencia en el espacio escolar, reduciendo de manera sustancial la desigualdad y la exclusión de niños y niñas, en especial de los más pequeños.

A partir de lo expuesto anteriormente, es prioritario formular las siguientes preguntas: ¿Qué entiende la infancia por participación?, ¿cuáles son los referentes que conforman su concepto de participación? Con la intención de dar respuesta a estos interrogantes, en el presente trabajo se analizarán las conceptualizaciones sobre participación de niños y niñas que cursan los primeros años escolares de educación primaria (primero y segundo grado) y que tienen entre cinco y ocho año. Asimismo, el uso y las prácticas que evocan sus profesores y familiares cuando utilizan la palabra participación, con el fin de identificar los referentes conceptuales y prácticos que van delineando su concepto de participar, para así evidenciar los principales obstáculos que enfrenta la construcción de escuelas inclusivas desde el entendimiento de las conceptualizaciones de los adultos y la niñez.

\section{Perspectiva teórica}

\section{La infancia en el terreno educativo: entre la protección y la exclusión}

El interés por estudiar a niños y niñas aumentó cuando comenzaron «a ser vistos como seres perfectibles y necesitados de educación» (Carrasco-Aguilar et al., 2016, p. 148). De ese modo, se reconoce a la infancia a partir de sus características propias, definidas 
por su situación (cuestiones permanentes en su vida: marginación, pobreza, entre otras) y condición (cuestiones temporales: edad, evolución de sus capacidades, entre otras). Los principales constructos teóricos que delimitan los marcos de acción de la infancia en la escuela se asocian a la psicología del desarrollo en relación con su evolución, esto es, derivados del estudio de su condición. A pesar de que estos planteamientos han permitido ordenar el proceso educativo, cabe señalar que los fundamentos de estas teorías son «criterios válidos para los adultos, lo cual conduce a destacar sus carencias, en vez de valorar lo que los niños pueden ofrecer» (Lansdown, 2005, p. 14).

$\mathrm{Al}$ percibir a los niños y las niñas como «personas que están en formación», es común que se espere poco o nada de la infancia; incluso en los discursos recientes sobre justicia social se menciona que «las capacidades de los niños de libre elección están inmaduras y son susceptibles de sucumbir a las presiones parentales para trabajar o dejar sus estudios» (Nussbaum, 2012, p. 185). Esta postura ejemplifica una visión evolucionista de la infancia, en la que la protección es el punto nodal del análisis.

Al respecto, Giroux (2003) refiere que la protección hacia la infancia establece relaciones específicas de poder que configuran las experiencias de los niños; en palabras del autor:

Se otorga a los niños el derecho a la protección, pero, al mismo tiempo, se les niega la capacidad de actuar y la autonomía. Incapaces de entender a la infancia como una interpretación histórica, social y política, entremezclada con las relaciones de poder, muchos adultos envuelven a los niños en un aura de inocencia y proteccionismo que elimina toda idea viable de responsabilidad adulta, aunque la evoque. (p. 14)

Las miradas de niños y niñas centradas en la protección se agudizan con niños y niñas de menor edad, lo que fundamenta mitos que restringen sus marcos de acción en la vida escolar. Algunos de estos son:

- El desarrollo infantil es un proceso universal.

- La adultez tiene estatus normativo.

- Los objetivos del desarrollo son universales.

- La desviación de la norma representa un peligro para el niño. (Lansdown, 2005, p. 10)

El proteccionismo se sustenta en el adultocentrismo, entendido como el «sistema de dominación que delimita accesos y clausuras a ciertos bienes, a partir de una concepción 
de tareas de desarrollo que a cada clase de edad le corresponderían» (Duarte, 2012, p. 111). Este es uno de los principales problemas que enfrenta la infancia, debido a que restringe la participación en los entornos educativos. Las prácticas adultocéntricas se sustentan en relaciones desiguales de poder entre profesores y estudiantes, gestadas en la protección de los niños y las niñas, lo que conduce a un camino de prohibiciones legitimado por la sociedad, que hace hincapié en el poco respeto por su expresión; por lo que los obstáculos para el ejercicio de su participación «no surgen de las inhabilidades, sino de su posicionamiento social» (Alderson \& Goodey, 1996, p. 106).

\section{Participación de la infancia}

La participación, a partir de la Convención sobre los Derechos del Niño (CDN), ha generado una reestructuración del posicionamiento del niño en la sociedad, pues les confiere a los niños y las niñas ser sujetos de derechos, que interactúan en su medio y, a su vez, modifican el lugar en el que se desenvuelven a partir de ser consultados en todo acontecimiento que los involucre.

Debido a la diferencia sustancial en su desarrollo físico y psicológico en la infancia, la CDN hace una distinción entre el nivel de injerencia que tienen sus opiniones según su etapa biológica, al tiempo que menciona que «deben ser tomadas en cuenta en función de la edad y madurez» (Unicef, 2016, p. 13); esto significa que, a medida que evolucionen sus capacidades, sus opiniones tendrán mayor peso (Eriksen, 2018). Al respecto, Moss (2007) critica esta perspectiva y destaca que la participación de los niños y las niñas más pequeños implica la formulación de diálogos que facilitan entender los numerosos lenguajes de expresión de la niñez, lo que permite «dar sentido» a conceptos técnicos de la democracia, como la participación, a partir de dar «voz» a los niños sobre sus construcciones conceptuales.

De esa manera, la participación conlleva posicionar a los niños y las niñas como interlocutores válidos que se expresan en múltiples formas. Ello que debería permitir su visibilidad, estar implicados en la toma de decisiones de los procesos que los involucren y ser reconocidos como personas con ideas, pensamientos e inquietudes, que dan cuenta de su individualidad; asimismo, destacar sus intereses y preocupaciones de participar activamente en la solución de problemas sociales y escolares como sujetos capaces de desempeñar un papel protagónico en sus espacios vitales (Serrano-Arenas \& OchoaCervantes, 2019). 
En los estudios sobre el ejercicio de la participación infantil se categoriza la clase de prácticas que los adultos les permiten a niños y niñas (Hanson, 2012; Hart, 1993; Malone \& Hartung, 2010; Santos, 2003; Trilla \& Novella, 2001). Así, los tipos de participación que coinciden son la participación simbólica, que consiste en que niños y niñas realizan acciones desde la heteronomía; es decir, participan en actividades definidas por los adultos, por lo que son ajenas a su reflexividad y conciencia. Igualmente, desconocen los fines de su participación, ya que esta responde al canon de la obediencia. El segundo tipo es la participación consultiva, que es cuando se toman en cuenta las opiniones de niños y niñas, aunque no transformen la estructura preestablecida por los adultos que los rodean. Finalmente, el tercer tipo es la participación plena o emancipadora, cuando niños y niñas realizan acciones desde su iniciativa y los adultos pueden colaborar en el desarrollo de las acciones.

Los primeros dos tipos de participación responden a relaciones verticales que mantienen una estructura jerárquica, en la que el proceso de aprendizaje está diseñado y dirigido por los adultos que los rodean, quienes lo consideran prioritario. Estas clases de participación no responden a la diversidad de realidades que coexisten en la escuela, debido a que casi todas las actividades se diseñan sin tomar en cuenta los pensamientos y las expresiones de los niños y las niñas. Por su parte, la tercera clase de participación exige el diseño y los métodos de intervención de actividades con propósitos de aprendizaje más abiertos, que dialoguen con las expresiones de los niños y las niñas en cuanto a sus necesidades e intereses; de ese modo, se posibilitaría el ejercicio de la participación plena.

Las prácticas tradicionales recaen en los dos primeros tipos de participación, que no abonan a la atención a la diversidad, sino que más bien promueven la exclusión de la infancia al limitar su participación. Dichas prácticas restrictivas pueden tener buenas intenciones, pero no dejan de ser excluyentes. Por esto, la participación, de manera imperativa, debe apuntar a un ejercicio de la participación plena, lo que permitiría construir escuelas centradas en las necesidades e intereses de los niños y las niñas.

\section{La participación como un eje en la construcción de escuelas inclusivas}

La inclusión se debe considerar como una búsqueda permanente de procedimientos, cada vez más adecuados, para responder a la diversidad, convivir con la diferencia y aprender a aprender de ella (Gutiérrez, Martín, \& Jenaro, 2014, p. 187). Por esto la escuela, como espacio inclusivo, exige reconocer a cada persona como un ser único y diferente de los otros, no para acentuar las diferencias, sino para localizar los puntos de encuentro que permitan atender la diversidad de las necesidades de cada persona a partir de fun- 
damentar el acto educativo en el común entendimiento entre niños, niñas, profesores y otros actores escolares.

El diálogo es una herramienta indispensable para identificar y plantear las necesidades de la gente involucrada, fuera de generar espacios para compartir expresiones, experiencias y reflexiones de su vida escolar (De Oña, García, \& Colomo, 2018). Es por ello que la participación es un eje fundamental de las escuelas inclusivas, al reducir la exclusión de los sujetos y al promver la expresión de las personas sobre sus necesidades, problemas e intereses.

No obstante, garantizar la participación en las escuelas no se limita solamente a la expresión y toma de decisiones, sino también a generar espacios y condiciones que permitan a las personas con riesgo de ser excluidas, como niños y niñas de menor edad, incrementar sus oportunidades de acción en los procesos educativos; esto es, involucrar a la infancia activamente en su aprendizaje y evaluación, así como en la solución de problemas relacionados con la convivencia escolar. La idea con esta participación de la infancia es ampliar los rangos de expresión y acción de la gente, lo que permitiría gestar relaciones de entendimiento ante la diferencia y diversidad.

\section{Método}

\section{Diseño}

La presente investigación tiene un enfoque cualitativo, de orden analítico-descriptivo. El método que se utilizó fue el cuasietnográfico (Goetz \& Lecompte, 1988), que permitió un acercamiento directo con los grupos de estudio utilizando diversas técnicas de recolección de datos y, con ello, conocer de primera mano las narraciones de los sujetos en su cotidianidad.

\section{Participantes}

La investigación se efectuó en tres escuelas de educación primaria ubicadas en entidades federativas del Estado de México y Ciudad de México, cuyas poblaciones pertenecen a comunidades urbanas-marginales; esto es, las escuelas se localizan en poblaciones en las que se concentran la pobreza y la marginación, por lo que tienen dificultad a la hora de satisfacer sus necesidades básicas. Es común que los cuidadores de niños y niñas 
trabajen amplias jornadas laborales y que, por ende, niños y niñas tengan responsabilidades de su cuidado, al igual que el de hermanos y hermanas de menor edad.

En cada escuela se consideró la participación de niños y niñas en un rango de edad entre cinco y ocho años (media de edad 6.7), por lo que el subgrupo de estudio fueron los niños y las niñas que se encontraban cursando primero y segundo año de educación primaria. En el estudio participaron 93 niñas y 87 niños, para una población total de 180 .

Con la intención de comprender los referentes de los niños y las niñas de estudio sobre los conceptos de participación, se entrevistó a un docente de primer grado y a un docente de segundo en cada escuela (para un total de seis), así como a veinticuatro cuidadores directos de niños y niñas participantes de primer año (ocho en cada escuela), y a dieciséis de segundo (cinco en cada escuela y una profesora que es madre de familia a su vez), para un total de cuarenta. Los cuidadores que participaron fueron aquellos que acudieron a la escuela cada vez que los investigadores los convocaron.

Los grupos escolares se tomaron de acuerdo con el criterio de selectividad en cuanto a los tiempos y los espacios en los que se generaron las narrativas (Goetz \& Le Compte, 1988) y el criterio de accesibilidad, determinado por la disponibilidad temporal y espacial que brindaron los actores escolares (Valles, 2000). El estudio se efectuó en estas escuelas debido al trabajo etnográfico realizado en investigaciones previas, lo cual facilitó la incursión en campo y el acceso a los informantes claves; estos últimos, como resultado de la cotidianidad, la convivencia y la interacción frecuente, expresaron sus pensamientos y opiniones respecto a los usos y conceptos de la participación en un ambiente de confianza y naturalidad comunicativa entre los sujetos y los investigadores.

Se invitó a participar en la investigación a profesores y familiares, niños y niñas de dichos grupos escolares, para que explicaran sus fines. Se les pidió firmar una carta de consentimiento como participantes de la investigación, haciendo de su conocimiento los objetivos, procesos, métodos y finalidades del trabajo académico.

\section{Instrumentos y procesamientos para la producción de datos}

El instrumento de recolección de información con profesores y familiares fue la entrevista abierta (Pérez, 2005), que permitía enfocar el diálogo en torno a la participación cuando se comunican con la infancia. Esta clase de entrevista se realizó con la finalidad de profundizar en los conceptos sobre participación y los elementos a los que aluden cuando utilizan esta palabra, con la intención de desentrañar los principales referentes 
que influyen en la conformación de las conceptualizaciones de participación en los niños y las niñas.

En el caso de los niños y las niñas - y tomando en cuenta su diversidad de lenguajes de expresión - se formularon tres instrumentos para la recolección de información sobre la conceptualización de la participación, mismos que se emplearon en el siguiente orden:

- Grupos focales detonados por diálogos horizontales.

- Entrevistas semiestructuradas.

- Dibujo sobre su concepto de participación.

Se armó un grupo focal, en el que se compartieron los resultados de la investigación que realizaron niños y niñas sobre el concepto de participación de sus compañeros, promoviendo de esa manera diálogos horizontales entre pares, que consistieron en que cuatro niños y niñas preguntaban a sus compañeros sobre el concepto durante el recreo escolar. Quienes manifestaron interés por entrevistar a sus compañeros fueron aquellos que participaron en dichos diálogos horizontales. Después de ello, se compartieron las respuestas obtenidas. Estos diálogos horizontales permitieron que niños y niñas se expresaran sin temor a equivocarse, evitando la complacencia a las personas adultas y la presión de dar una respuesta, propiciando un ambiente de confianza en paridad.

Las entrevistas se hicieron en forma individual para averiguar cuáles son los principales referentes a los que aluden cuando escuchan la palabra. Por último, se les solicitó realizar un dibujo, cuya estrategia permite identificar los referentes y marcos interpretativos de los sujetos de estudio (Canales, 2006) sobre lo que consideran es participación y los lugares principales donde participan, acompañado de una explicación individual sobre objetos y lugares que dibujaron.

\section{Procesamiento y análisis de datos}

El procesamiento de los datos se hizo por medio del análisis de contenido, considerando en primer momento la codificación abierta, la cual permite generar comprensiones e interpretaciones socioculturales al contenido de las palabras; posteriormente, se realizó la codificación axial, que consistió en hacer la indagación de preguntas claves para generar redes de relaciones entre las palabras (qué, dónde y con quiénes participan), lo que llevó a la generación de las categorías conceptuales (Strauss \& Corbin, 2002). 
El análisis de datos recopilados en las entrevistas y los grupos focales se documentaron digitalmente en formato audio, para su posterior transcripción. De la información de las transcripciones e interpretaciones de los dibujos se elaboraron «códigos en vivo» (Strauss \& Corbin, 2002); es decir, se agrupó la información en códigos conceptuales, lo que permitió resguardar las expresiones de la población de estudio.

\section{Resultados}

\section{Conceptualizaciones de niños y niñas sobre participación}

Los resultados se presentan organizados en torno a tres categorías fundamentales, derivadas del análisis de datos: la participación como ayuda, la participación como actividad corporal y la participación como trabajo académico. Estas tres categorías proporcionaron la base de un marco conceptual sobre la participación, expresado por niños y niñas entre cinco y ocho años del sistema escolar de nivel primario.

Las conceptualizaciones sobre participación difirieron entre niños y niñas de siete y ocho años (segundo grado), con los de cinco y seis años (primer grado), pues los más pequeños mostraron dificultad para definir actividades relacionadas con el concepto de participación. Esta situación se hizo latente en los diálogos horizontales, ya que sus respuestas mostraron tener escasos referentes de la palabra participar. Así lo ejemplifican los siguientes fragmentos:

Entrevistador (niño de cinco años): —¿Qué piensas que es participación?

Entrevistados: —Nada. (niña de cinco años)

-No sé. (niña de seis años)

-Ir a Francia. (niño de cinco años)

-Para mí, es querer mucho la escuela. (niño de seis años)

\section{La participación como ayuda}

Los significados de participación relacionados con ayudar se remitieron a tres espacios de acción: el familiar, el escolar y el comunitario. En el primero, los niños y las niñas mencionaron que participan cuando ayudan en los quehaceres de la casa, mientras que en el espacio escolar niños y niñas consideraron que participar es ayudar al profesorado con aquellos niños con dificultad para aprender o seguir las normas establecidas en el aula escolar. Expresiones que dan cuenta de lo anterior son: 
Ayudo levantando los zapatos, también le ayudo a mi mamá con el quehacer. (niño de seis años)

Participar es decir a la maestra si unos niños se están peleando. (niña de siete años)

Participo cuando hago lo que me dicen que haga. (niña de seis años)

Participar es ayudar a recoger la basura en el salón. (niño de seis años)

Derivado de la indagación a sus respuestas, niños y niñas manifestaron que no determinan las actividades en las que ayudan, sino que más bien estas son asignadas por sus profesores y familiares. De ese modo, los significados de participación relacionados con ayudar en los espacios escolar y familiar están dirigidos a la obediencia de la infancia a los adultos con quienes interactúan en un espacio en concreto. A diferencia de estos espacios, en el comunitario las formas de ayuda no se remitieron a obedecer a los adultos, sino en hacer donaciones a los desfavorecidos, desprotegidos o a personas en situaciones de crisis y desventura, por lo que la ayuda a la que hacen referencia es de tipo asistencialista. Expresiones que enmarcan lo anterior fueron:

La participación es dar cobijas a los pobres. (niña de siete años)

Las personas participan cuando dan ayuda en el temblor. (niño de seis años)

Vale la pena señalar que los niños de cinco y seis años destacaron la participación en el ámbito comunitario, mientras que los niños de siete y ocho años se remitieron, principalmente, al ejercicio de su participación en el ámbito escolar.

\section{La participación como actividad corporal}

Las prácticas participativas en esta categoría evocaron el espacio escolar como el principal escenario de acción; destacaron al juego, los concursos y las competiciones como las principales manifestaciones de su participación. Estas declaraciones, expuestas en las entrevistas, se corroboraron en los dibujos realizados sobre el ejercicio de su participación que aludieron a actividades lúdicas como jugar baloncesto, fútbol, hacer carreras entre compañeros, entre otras actividades de carácter físico. Cuando se preguntó sobre las razones de su dibujo, niños y niñas expresaron que participan cuando juegan o hacen ejercicios. Ejemplo de ello son los siguientes diálogos:

Yo participo cuando hago ejercicios. (niña de seis años) 
Cuando jugamos en educación física. (niña de siete años)

Participar es echar carreritas. (niño de seis años)

Los niños y niñas entre cinco y seis años de edad con frecuencia mostraron dificultad para expresar significados o prácticas que relacionaban con la participación; después de unos momentos, y al decirles que podían pronunciar cualquier palabra que pensaran que tuviera que ver con la participación, evocaron actividades prácticas, como pintar, bailar, correr y hacer ejercicio. Estas prácticas relacionadas con la actividad corporal fueron destacadas principalmente por niños y niñas de primero de primaria, es decir, menores de entre cinco y seis años de edad, por lo que se puede concluir que la participación la relacionan con experiencias corporales.

\section{La participación como trabajo académico}

Los niños y las niñas de estudio relacionaron la participación con actividades académicas tales como el acto de aprender, realizar las tareas de la escuela, trabajar en el salón de clases, seguir las reglas del aula, alzar la mano y trabajar en equipo. Los diálogos y dibujos de los niños entre siete y ocho años se relacionaron, principalmente, con el acto de emitir una opinión cuando les piden participar (alzar la mano) y cumplir con las tareas solicitadas por el profesor, mientras que entre los menores de cinco y seis años fueron una minoría quienes asociaron la participación con la práctica de expresar sus opiniones cuando levantan la mano en el aula escolar. Algunas concepciones manifestadas por niños y niñas fueron:

Yo pienso que participar es lo que tengo que decir para poder sacar diez; participo cuando alzo la mano y digo la respuesta correcta. (niño de siete años)

Participar es decir lo que el maestro quiere escuchar. (niña de ocho años)

De esa manera, la participación se asocia con realizar tareas escolares o exponer una opinión en el aula escolar; estas tareas evocan la complacencia a sus profesores.

\section{La perspectiva adulta sobre la participación de la infancia}

Los adultos entrevistados fueron los profesores de grupo y familiares de los niños y las niñas, al ser las personas con mayor tiempo de interacción con ellos y ellas en su cotidianidad. En las entrevistas se indagó sobre los significados de la participación, las formas en que niños y niñas ejercen su participación, así como acerca de los usos de la 
palabra en la cotidianidad. La información proporcionada en la entrevista versó sobre las siguientes categorías:

\section{La participación como derecho}

El cuerpo docente identifica la participación como un derecho a ser escuchado y permitir la expresión en el salón de clases. Las principales manifestaciones de la participación desde esta perspectiva aluden a la expresión de la infancia cuando les piden su punto de vista sobre un aprendizaje o situación dentro del aula; asimismo, todo el profesorado considera que niños y niñas participan cuando levantan la mano para expresar sus saberes previos o se les pregunta sobre una situación, es decir, cuando dan su opinión en asuntos que se les consultan. Por otro lado, el cuerpo docente da cuenta de que existen dos tipos de participación: la positiva y la negativa. La primera es cuando niños y niñas hacen las cosas adecuadas (cuando las solicita el profesor), mientras que la participación negativa da cuenta de los estudiantes que organizan actividades con otros compañeros que no siguen las normas escolares o atentan contra su bienestar. Los siguientes fragmentos son una prueba de lo anterior:

La participación es un derecho que tienen los niños cuando expresan lo que sienten, lo que piensan y lo que viven; los niños participan cuando alzan la manita o se acercan al escritorio para decirte algo... Los niños participan cuando comparten sus ideas, cuando hacen las cosas bien, cuando cumplen con sus actividades, cuando ayudan a sus compañeros; si no, es una participación negativa. (profesora de segundo año)

A su vez, tanto los profesores como las profesoras expresaron que, cuando usan la palabra participar, esperan que los niños y las niñas se involucren y expresen sus puntos de vista; en este sentido, el concepto de participación se utiliza principalmente para que niños y niñas emitan una opinión sobre un aspecto de la vida escolar cuando se les solicita, así como respetar las normas establecidas en la escuela.

\section{La participación como ayuda}

Tanto el profesorado como familiares relacionaron la participación con ayudar en el desarrollo de actividades de la vida cotidiana. Los profesores manifiestan que alumnos y alumnas participan cuando trabajan en equipo y, a su vez, consideran que palabras que promueven la participación de los niños son apoyar, ayudar, colaborar, hacer, entre otras. 
En el caso de los familiares, en su mayoría manifiestan que niños y niñas participan cuando ayudan en las tareas de la casa, y también expresan que realizan quehaceres en casa cuando ellos (los adultos) se los asignan y deben obedecer. Por otro lado, manifiestan que, en las actividades de esparcimiento social, niños y niñas participan en el desarrollo de las actividades, pero pocas veces tienen injerencia en la selección de estas. Los adultos afirmaron al respecto:

Participar es ayudar... contribución... cosas que se trabajan entre todos. (madre de niño de primer año)

La participación es colaboración que se da a una o varias tareas. (abuelita de niña de ocho años)

Participar es ayudar; él es muy participativo: recoge sus zapatos hace el quehacer en casa, tiene que recoger los platos. (mamá de niño de siete años)

Cabe destacar que todos los familiares manifestaron que emplearon la palabra participar para asignar tareas o en la convivencia cotidiana con los niños y las niñas, pero las expresiones que ellos utilizan que tienen que ver con la participación son ayudar, hacer y realizar. Algunas expresiones en torno a ello fueron:

Entrevistador: - ¿Usa la palabra participar con su hijo/a?

Entrevistados: -No, uso la palabra hacer cuando quiero que hagan cosas. (mamá de niña de siete años)

-No, no tenemos la costumbre de decir participar, digo hago o ayúdame. (madre de niño de seis años)

-No, pero sí participa. (papá de niña de siete años)

\section{Discusión}

Como se ha indicado con anterioridad, el objetivo de la presente investigación consiste en identificar las concepciones sobre participación que tienen niños y niñas en sus primeros años escolares en la educación primaria, así como los referentes que influyen en su construcción. Las conceptualizaciones de niños y niñas más pequeños, entre cinco y seis años de edad, se concentran en actividades relacionadas con el movimiento corporal y en ayudar a sus compañeros, profesores y familiares, mientras que los niños y las niñas con un año más de escolaridad relacionan el ejercicio de su participación con acti- 
vidades que les son dictadas y en emitir una opinión cuando es solicitada por sus profesores en la escuela; ello a diferencia de los más pequeños, que en escasas ocasiones mencionaron esta práctica como un referente de su participación.

Relacionado con lo anterior, la totalidad de profesores entrevistados consideran que los niños y las niñas ejercen su participación cuando levantan la mano para expresar su opinión y, en contraste con esto, sus familiares declararon no usar la palabra participación en espacios de interacción con los niños y las niñas. Lo anterior demuestra que conforme la infancia avanza en su escolaridad (institucionalización), los conceptos sobre su participación se van delineando en que tiene que ver con sus profesores. Esto pone en evidencia una estructura escolar que reduce la participación de niños y niñas a los imperativos de los adultos, de manera que las escuelas se construyen desde perspectivas adultocéntricas que pocas veces dialogan con la infancia en la construcción del espacio escolar.

Lo anterior coincide con los hallazgos de Ochoa-Cervantes (2015), quien afirma que las concepciones de los niños y las niñas entre ocho y doce años de edad refieren a la práctica de emitir una opinión en el espacio escolar como la principal forma de ejercer su participación. Por lo anterior, el presente estudio permite identificar el principal obstáculo en la construcción del concepto de participación de la infancia: su enseñanza de desde una posición de obediencia.

$\mathrm{Al}$ ser la obediencia el fundamento de las relaciones entre la infancia y los adultos, el adultocentrismo se encuentra presente en las relaciones escolares entre profesores y niños y niñas, impidiendo la construcción de conceptualizaciones de participación más amplias e incluyentes y, por tanto, reduciendo las prácticas participativas a una relación de poder y subordinación de los niños y las niñas a los referentes conceptuales y procedimentales preestablecidos en las instituciones escolares (Carrasco-Aguilar et al., 2016). Referentes estos que son producto de las prácticas participativas que les son permitidas, ya que se estructuran «lateralmente al lenguaje de la acción» (Foucault, 2014, p. 61); esto significa que las conceptualizaciones construidas recaen en las formas en que ejercen su participación y en las prácticas que limitan o expanden los marcos de acción de la infancia.

Por esto se hace indispensable incluir a los niños y las niñas como copartícipes plenos de la vida escolar, capaces de interactuar con profesores y familiares en situaciones igualitarias; es decir, «desinstitucionalizar los patrones de valor cultural que impiden la paridad de participación y remplazarlos por patrones que la favorezcan» (Fraiser, 2006, p. 37). 
A partir de los hallazgos expuestos, se recomienda el diálogo entre los significados de la participación entre profesores, familiares, niños y niñas para una construcción reflexiva del concepto con mayor sentido para la infancia, lo que implicaría ampliar los significados de la participación, pues «la expresión o expresiones que la describen en la lengua local (...) adquiere un significado más específico» (Liebel \& Saadi, 2012, p. 125) al asociarlo con sus referentes en el ámbito sociocultural (Arcos-Miranda, Serrano-Arenas, \& Ochoa-Cervantes, 2019).

Así las cosas, estos diálogos generados no solamente impactarían en las conceptualizaciones de los niños y las niñas, sino también en la ampliación de los espacios que promuevan las expresiones de la infancia y prácticas participativas horizontales entre adultos y la niñez. Lo anterior permitiría la construcción de escuelas inclusivas al tomar los referentes «sociales y morales, las creencias y sus conexiones con las políticas y las prácticas en las escuelas (...) en la compleja interacción entre individuos y grupos» (Gutiérrez, Martín, \& Jenaro, 2014), coadyuvando en la inclusión de la infancia en el espacio educativo.

La reflexión y el consenso de los fines de la participación que se pretende promover con la infancia evitarían prácticas restrictivas de la participación, así como también ayudarían a que niños y niñas identifiquen con mayor facilidad las formas en que la ejercen en los ámbitos familiar y comunitario, lo que llevaría a la construcción de «escenarios para la participación de ellas y ellos» (Gallego-Henao, 2015, p. 156).

Cabe destacar que a pesar de los esfuerzos por identificar los referentes de la participación de niños y niñas en sus primeros años de educación primaria, en el presente estudio no se profundizó en los referentes conceptuales de los profesores provenientes de su currículo escolar. Hacerlo permitiría entender las razones institucionales que sustentan las relaciones de subordinación de la infancia a la estructura escolar preestablecida, siendo esta un área de oportunidad de estudios posteriores en este campo del conocimiento. Dichas acciones conformarían un avance sustancial en una participación plena de la infancia que se aleje de la adaptación de la infancia a la estructura escolar y promueva su inclusión en la construcción de la vida escolar.

\section{Agradecimientos}

Al Consejo Nacional de Ciencia y Tecnología, por su apoyo para realizar la estancia posdoctoral. 


\section{Referencias}

Ainscow, M. (2012). Haciendo que las escuelas sean más inclusivas: lecciones a partir del análisis de la investigación internacional. Revista de Educación Inclusiva, 5(1), 39-49.

Albornoz, N., Silva, N., \& López, M. (2015). Escuchando a los niños: significados sobre aprendizaje y participación como ejes centrales de los procesos de inclusión educativa en un estudio en escuelas públicas en Chile. Estudios Pedagógicos, 41(especial), 81-96. http://dx.doi.org/10.4067/So718-07052015000300006

Alderson, P. (2015). Justice, rights, agency and childhood research. The International Journal of Children's Rights, 23, 667-676. https://doi.org/10.1163/15718182-02303013

Alderson, P., \& Goodey, C. (1996). Research with disabled children: How useful is childcentred ethics? Children \& Society, 10(2), 106-116. https://doi.org/10.1111/chso. 1996.10.2.106

Ancheta-Arrabal, A. A. (2015). Repensando las voces de la primera infancia en la era global. Espacios en Blanco. Serie Indagaciones, 25(1), 21-40.

Ancheta-Arrabal, A. A. (2019). Equidad y educación de la primera infancia en la agenda educativa mundial. Revista Latinoamericana de Ciencias Sociales, Niñez y Juventud, 17(1), 47-59.

Arce, M. C. (2015). Hacia un discurso emancipador de los derechos de las niñas y los niños. Lima: Ifejant.

Arcos-Miranda, E., Serrano-Arenas, D., \& Echeverría, G. M. R. (2019). La enseñanza de la historia en el medio indígena: del olvido social a la memoria colectiva. Ciudad de México: Castellanos Editores.

Canales, M. (2006). Metodologías de la investigación social. Santiago: Lom.

Carrasco-Aguilar, C. L., Verdejo-Valenzuela, T., Asun-Salazar, D., Álvarez, J. P., BustosRaggi, C., Ortiz-Mallegas ... Valdivia-Hennig, N. (2016). Concepciones de infancia en una escuela con altos índices de violencia escolar de estudiantes a profesores. Revista Latinoamericana de Ciencias Sociales, Niñez y Juventud, 14(2), 1145-1159. https://doi.org/10.1160o/1692715x.14218210814

Cubillos, G. M. F., Chiappe, S. M. D., González, J. M. P., \& Velásquez, E. L. C. (2018). Creencias sobre educación inicial en colegios oficiales de Bogotá. Infancias Imágenes, 17(1), 100-108.

De Oña, C. J. M., García, E. A. G., \& Colomo, M. E. (2018). Educación de adultos e inclusión social. Experiencias y opiniones de un grupo de estudiantes. Revista 
Internacional de Educación para la Justicia Social, 7(2), 1-17. https://doi.org/10.15366/ riejs2018.7.2.006

Díaz-Bórquez, D., Contreras-Shats, N., \& Bozo-Carrillo, N. (2018). Participación infantil como aproximación a la democracia: desafíos de la experiencia chilena. Revista Latinoamericana de Ciencias Sociales, Niñez y Juventud, 16(1), 101-113. https://doi.org/ $10.11600 / 1692715 \times .16105$

Duarte, C. (2012). Sociedades adultocéntricas: sobre sus orígenes y reproducción. Revista Última Década, 20(36), 99-125. http://dx.doi.org/10.4067/So718-22362012000100005

Eriksen, E. (2018). Democratic participation in early childhood education and careserving the best interests of the child. Tidsskrift for Nordisk barnehageforskning, ${ }_{17}(1)$, 1-15. https://doi.org/10.7577/nbf.2319

Foucault, M. (2014). Las palabras y las cosas: una arqueología de la ciencia. Ciudad de México: Siglo XXI.

Fraiser, N. (2006). ¿De la redistribución al reconocimiento? Madrid: Morata.

Frasco-Zuker, L. (2016). Investigación etnográfica sobre experiencias de trabajo infantil en el noreste argentino. Revista Latinoamericana de Ciencias Sociales, Niñez y Juventud, 14(2), 1205-1216. https://doi.org/10.11600/1692715X.1417090915

Gallego-Henao, A. M. (2015). Participación infantil... Historia de una relación de invisibilidad. Revista Latinoamericana de Ciencias Sociales, Niñez y Juventud, 13(1), 151-165. https://doi.org/10.1160o/1692715x.1318060514

Gallego-Henao, A. M., \& Gutiérrez-Suárez, D. (2015). Concepciones adultas sobre participación infantil en relación con la toma de decisiones de los niños. Zona Próxima, (22), 87-104. https://doi.org/10.14482/zp.22.6078

Gascón, F., \& Godoy, L. (2015). Presencia e in-diferencia: por un estatuto visual de la niñez. Revista Latinoamericana de Ciencias Sociales, Niñez y Juventud, 13(2), 645-656. https://doi.org/10.11600/1692715x.1326093014

Giroux, A. H. (2003). La inocencia robada: juventud, multinacionales y política cultural. Madrid: Morata.

Goetz, J., \& LeCompte, M. (1988). Etnografía y diseño cualitativo en investigación educativa. Madrid: Morata.

Gutiérrez, M. O., Martín, V. C., \& Jenaro, R. C. (2014). El índex para la inclusión: presencia, aprendizaje y participación. Revista de Educación Inclusiva, 7(3). 186-201. Hanson, K. (2012). Schools of thought in children's rights. En M. Liebel et al. (eds.), Children's rights from below: Cross-cultural perspectives (pp. 63-79). Basingstoke: Palgrave Macmillan. https://doi.org/10.1057/9780230361843-5 
Hart, R. (1993). Children's participation: From tokenism to citizenship. Florencia: Unicef.

Hedrera-Manara, L., \& Álvarez-Martínez, C. (2018). Los niños y las niñas recuerdan: memoria colectiva y participación en Memorial Paine, Chile. Revista Latinoamericana de Ciencias Sociales, Niñez y Juventud, 16(2), 961-977. https://doi.org/10.1160o/1692715x.16221

Lansdown, G. (2005). La evolución de las facultades del niño. Florencia: Unicef, Innocenti Research Centre.

Leyra-Fatou, B., \& Bárcenas-Viñas, A. M. (2014). Reflexiones etnográficas sobre el ocio infantil. Revista Latinoamericana de Metodología de las Ciencias Sociales, 4(1), 1-21.

Liebel, M. (2015). Sobre el interés superior de los niños y la evolución de las facultades. Anales de la Cátedra Francisco Suárez, 49, 43-61.

Liebel, M., \& Invernizzi, A. (2018). Los movimientos de niños, niñas y adolescentes trabajadores y la Organización Internacional del Trabajo: una lección sobre el silencio forzado. Millcayac, Revista Digital de Ciencias Sociales, 5 (8), 89-112.

Liebel, M., \& Saadi, I. (2012). La participación infantil ante el desafío de la diversidad cultural. Antropos, 39, 33-44. https://doi.org/10.29340/39.244

Malone, K. \& Hartung, C. (2010). Challenges of participatory practices with children. En B. Percy-Smith, \& N. Thomas (Eds.), A handbook of children and young people's participation: Perspectives from theory and practice (pp. 24-38). Nueva York: Routledge. https://doi.org/10.4324/9780203871072

Martínez-Núñez, M. D., \& Muñoz-Zamora, G. (2015). Construcción de imaginarios de la infancia y formación de educadoras de párvulos. Revista Latinoamericana de Ciencias Sociales, Niñez y Juventud, 13(1), 343-355. https://doi.org/10.1160o/1692715x.13120190814

Mendia, R. G. (2017). El aprendizaje-servicio como una estrategia inclusiva para superar las barreras al aprendizaje y a la participación. Revista de Educación Inclusiva, 5(1), 71-82.

Miranda, A., \& Albarrán, B. (2019). Crecer y soñar entre palabras: la lectura y la escritura en contextos desprovistos de modelos lectores. En Leer y escribir en contextos críticos: una experiencia de literacidad entre chicos y grandes (pp. 13-26). Ciudad de México: Castellanos Editores.

Moreno-Roldán, M. R., Agudelo-Bedoya, M. E., \& Alzate-Pulgarín, V. (2018). Voces a escuchar en el cuidado: ¿qué dicen los niños y las niñas? Revista Latinoamericana de Ciencias Sociales, Niñez y/uventud, 16(1), 227-237. https://doi.org/10.1160o/1692715x. 16113

Moss, P. (2007). Bringing politics into the nursery: Early childhood education as a democratic practice. European Early Childhood Education Research Journal, 15(1), 5-20. https://doi.org/10.1080/13502930601046620 
Nussbaum, M. (2012). Crear capacidades: propuesta para el desarrollo humano. Barcelona: Paidós.

Ochoa-Cervantes, A. (2015). Concepciones sobre participación de niñas, niños y adolescentes: su importancia en la construcción de la convivencia escolar. Cultura, Educación y Sociedad, 6(2), 9-28.

Patiño, G., \& Danna, L. (2017). Conocimiento y concepción de los compromisos pedagógicos para la primera infancia, de los profesores de Licenciatura en Pedagogía Infantil de la Universidad de la Sabana. (Tesis). Universidad de la Sabana, Bogotá, D. C., Colombia.

Pérez, F. (2005). La entrevista como técnica de investigación social: fundamentos teóricos, técnicos y metodológicos. Extramuros, 8(22), 187-210.

Santos, M. (2003). Participar es aprender a convivir. En M. Santos (Coord.), Aprender a convivir en la escuela (27-39). Madrid: Akal.

Serrano-Arenas, D., \& Ochoa-Cervantes, A. (2019). El aprendizaje servicio como potenciador de la educación inclusiva en la educación primaria. Revista Iberoamericana de Aprendizaje y Servicio. Ridas (7), 37-54.

Stoecklin, D. (2013). Theories of action in the field of child participation: In search of explicit frameworks. Childhood, 20(4), 443-457. https://doi.org/ 10.1177/0907568212466901

Strauss, A., \& Corbin, J. (2002). Bases de la investigación cualitativa. Medellín: Editorial de la Universidad de Antioquia.

Thomas, N., Graham, A., Powell, M. A., \& Fitzgerald, R. (2016). Conceptualisations of children's wellbeing at school: The contribution of recognition theory. Childhood, 23(4), 506-520. https://doi.org/10.1177/0907568215622802

Trilla, J., \& Novella, A. (2001). Educación y participación social en la infancia. Revista Iberoamericana de Educación, 26, 137-164.

Unicef. (2016). Convención sobre los Derechos del Niño. Madrid: Unicef.

Valle, A. R. (2018). Participación de niños indígenas mazahuas en la organización familiar del trabajo. Papeles de Trabajo sobre Cultura, Educación y Desarrollo Humano, $8(1), 1-11$.

Valles, M. S. (200o). Técnicas cualitativas de investigación social. Madrid: Síntesis.

Voltarelli, M. A. (2017). Los temas del protagonismo y la participación infantil en las producciones suramericanas. Revista Latinoamericana de Ciencias Sociales, Niñez y Juventud, 16(2), 741-756. https://doi.org/10.1160o/1692715x.16207 